\title{
IgG Autoantibodies Against Desmocollin 3 in Pemphigus Sera Induce Loss of Keratinocyte Adhesion
}

\author{
David Rafei, ${ }^{*}$ Ralf Müller, ${ }^{*}$ Norito Ishii, ${ }^{\dagger}$ \\ Maria Llamazares, ${ }^{*}$ Takashi Hashimoto, ${ }^{\dagger}$ \\ Michael Hertl, ${ }^{*}$ and Rüdiger Eming ${ }^{*}$ \\ From the Department of Dermatology and Allergology,* Philipps \\ University, Marburg, Germany; and the Department of Dermatology, ${ }^{\dagger}$ \\ Kurume University School of Medicine, Fukuoka, Japan
}

Pemphigus is considered an autoimmune bullous skin disorder associated with IgG against the desmosomal components, desmoglein 3 (Dsg3) and desmoglein 1 (Dsg1). This concept is supported by the in vitro and in vivo pathogenicity of anti-Dsg3/Dsg1 IgG and the mucosal blistering phenotype of mice with a genetic deficiency of Dsg3. Mice deficient for another desmosomal adhesion molecule, desmocollin 3 (Dsc3), show a similar pemphigus phenotype, and we investigated the pathogenicity of Dsc3-reactive IgG autoantibodies that were identified previously in a subset of patients with atypical pemphigus. We here demonstrate that IgG against Dsc 3 causes loss of adhesion of epidermal keratinocytes. Specifically, IgG against Dsc3 was purified from Dsc3-reactive pemphigus sera by affinity column chromatography using recombinant human Dsc3. Affinity purified IgG was functionally active and did not only react with recombinant Dsc3 but also with epidermis and cultured human keratinocytes. Moreover, Dsc3-reactive IgG induced loss of adhesion of epidermal keratinocytes in a dispase-based keratinocyte dissociation assay that was reversed on pre-adsorption with human Dsc3 but not Dsg3. These findings demonstrate that IgG autoantibodies against an additional component of the desmosomes, Dsc3, induce loss of keratinocyte adhesion and thus may contribute to blister formation in pemphigus. (Am J Pathol 2011, 178:718-723; DOI: 10.1016/j.ajpath.2010.10.016)

Pemphigus is a severe autoimmune bullous disorder of mucous membranes and skin which has been associated with autoantibodies (autoab) of the IgG class against two desmosomal components of epidermal keratinocytes, desmoglein 1 (Dsg 1) and desmoglein 3 (Dsg3). ${ }^{1,2}$ Distinct clinical pemphigus variants, such as pemphigus vulgaris (PV) and pemphigus foliaceus (PF) have been linked to the presence of IgG reactive with their specific target antigens, Dsg3 and Dsg1, respectively. ${ }^{3}$ Moreover, the clinical phenotype of these pem- phigus variants can be largely explained by the tissue expression pattern of their autoantigens. ${ }^{4} \mathrm{PV}$ is characterized by suprabasal loss of adhesion of the mucous membranes, the site where Dsg3 is predominantly expressed. ${ }^{5}$ In contrast, PF is recognized by superficial blisters of the skin but not of the mucosa because Dsg1 is preferentially produced by the subcorneal epidermis of stratified epithelia such as the skin.

In addition to the presence of four desmosomal Dsg isoforms, ie, Dsg1-4, epidermal desmosomes contain three different isoforms of another group of cadherins, namely desmocollin 1 (Dsc 1), desmocollin 2 (Dsc 2), and desmocollin 3 (Dsc 3). ${ }^{6,7}$ Dsc1 has been previously identified as the target antigen of IgA autoab in the subcorneal pustular dermatosis type of IgA pemphigus. ${ }^{8}$ Moreover, IgG autoab against Dsc1, Dsc2, or Dsc3 were detected in the sera of patients with paraneoplastic pemphigus and, occasionally, in patients with atypical pemphigus. ${ }^{9-12}$ However, the pathogenic relevance of Dscspecific autoab in these disorders remains unclear.

The concept that the pathogenesis of pemphigus is exclusively linked to IgG autoab against Dsg1 and Dsg3 has been challenged by the recent observation that mice with a genetic deficiency of Dsc3 show a pronounced blistering phenotype with suprabasal loss of epidermal adhesion that is highly reminiscent of $P V .{ }^{13}$ It has been reported that Dsc3, as Dsg3, is preferentially expressed in the basal and suprabasal layers of human epidermis the site where loss of adhesion occurs in PV. ${ }^{6,7,13}$ Dsg1 and Dsg3 not only interact in desmosomes via homophilic transinteraction, but presumably also interact by heterophilic binding with Dsc isoforms such as Dsc1 and Dsc3. However, an heterophilic transinteraction between Dsg3 and Dsc3 is unlikely based on recent in vitro findings. ${ }^{14}$

Supported by the German Research Council (Deutsche Forschungsgemeinschaft, He1602/8-1; 8-2 to MH) through the Coordination Theme 1 (Health) of the European Community's FP7, Grant agreement number HEALTH-F2- 2008-200515 (M.H.); and by the Federal Ministry for Education and Research (BMBF; Nr. 01 GN0973 to M.H. and R.E.).

M.H. and R.E. contributed equally to this work.

Accepted for publication October 21, 2010.

Current address of R.M.: Department of Dermatology, Venerology and Allergology, University of Lübeck, Germany.

Address reprint requests to: Rüdiger Eming, M.D., or Michael Hertl, M.D., Ph.D., Department of Dermatology and Allergology, Philipps University, Deutschhausstrasse 9, D-35033 Marburg, Germany. E-mail: eming@med. uni-marburg.de or hert|@med.uni-marburg.de. 
Table 1. Autoantibody Profile and Clinical Characteristics of the Studied Pemphigus Patients

\begin{tabular}{|c|c|c|c|c|c|c|c|c|}
\hline \multirow[b]{2}{*}{ Patient } & \multirow[b]{2}{*}{ Autoab isotype } & \multirow[b]{2}{*}{ Diagnosis } & \multirow[b]{2}{*}{ Clinical characteristics } & \multicolumn{5}{|c|}{ Desmosomal target antigens ${ }^{\star}$} \\
\hline & & & & Dsc1 & Dsc2 & Dsc3 & Dsg1 & Dsg3 \\
\hline 1 & $\lg G$ & Pemphigus vegetans & $\begin{array}{l}\text { Exophytic skin erosions and } \\
\text { oral erosions }\end{array}$ & - & - & + & - & - \\
\hline 2 & $\lg G$ & Pemphigus vegetans & $\begin{array}{l}\text { Hypertrophic verrucous } \\
\text { plaques with pustules and } \\
\text { erosions on foot, groin, and } \\
\text { scalp }\end{array}$ & - & - & + & + & - \\
\hline 3 & $\lg G$ & Pemphigus herpetiformis & $\begin{array}{l}\text { Multiple pustules, erythematous } \\
\text { herpetiform erosions, oral } \\
\text { erosions }\end{array}$ & - & - & + & - & - \\
\hline 4 & $\lg G$ & Pemphigus herpetiformis & $\begin{array}{l}\text { Erythematous cutaneous } \\
\text { erosions }\end{array}$ & - & - & + & - & - \\
\hline
\end{tabular}

Autoab, autoantibody; Dsc, desmocollin; Dsg, desmoglein.

*Serum IgG reactivity as determined by immunoblot analysis using recombinant Dsg and Dsc proteins (see Figure 2).

In the present study, affinity-purified, Dsc3-reactive IgG fractions from pemphigus sera induced loss of epidermal keratinocyte adhesion in a similar manner as pathogenic antiDsg3 IgG autoab. Thus, Dsc3 can be considered as a relevant autoantigen of pemphigus and Dsc3-reactive IgG autoab may contribute to the blistering phenotype of pemphigus.

\section{Materials and Methods}

\section{Patients}

Sera of four patients with atypical pemphigus (pemphigus vegetans and pemphigus herpetiformis) were investigated. Assessment of the clinical conditions revealed exophytic skin erosions of the fingers and oral erosions (patient 1), hypertrophic vegetative (verrucous) plaques with pustules and erosions on the foot, groin, and scalp (patient 2), widespread erythema, pustules, and erosions of the oral mucosa (patient 3) and multiple erythematous erosions on trunk and extremities (patient 4). The clinical diagnosis of pemphigus was confirmed by: (a) histopathological evidence of intraepidermal loss of adhesion; (b) IgG deposits on the surface of epidermal keratinocytes of perilesional skin by direct immunofluorescence; and (c) cell surface IgG reactivity on monkey esophagus epithelium by indirect immunofluorescence. The clinical and immunoserological characteristics of the patients are shown in Table 1. Except for patient 2, who also had Dsg1-reactive IgG, all of the other patients predominantly demonstrated IgG reactivity to Dsc3. Figure 1 depicts representative clinical pictures of the studied patients. All patients gave written consent to participate in this investigation, which adhered to the Declaration of Helsinki Guidelines and was approved by the local ethics committee.

\section{Recombinant Dsc and Dsg Proteins}

The recombinant Dsc and Dsg proteins were expressed in insect cells (High Five; Invitrogen, Carlsbad, CA) by infection with recombinant baculovirus as previously described ${ }^{9,15}$ (Figure 2A). Culture supernatants of infected cells were collected after 3 days and recombinant proteins were purified by affinity chromatography using nickel-nitrilotriacetic-linked agarose beads (Qiagen, Hilden, Germany) according to the manufac- turer's instructions. Purified proteins were gradually dialyzed against phosphate-buffered saline (PBS) supplemented with $0.5 \mathrm{mmol} / \mathrm{L} \mathrm{CaCl}_{2}$ and stored at $-20^{\circ} \mathrm{C}$. By immunoblot analysis, the purified recombinant proteins displayed the expected size and were specifically immunoreactive using a monoclonal mouse anti-Etag antibody (1:1000; Amersham, Biosciences, Uppsala, Sweden) (Figure 2B, upper panel). In addition, serum of a patient with paraneoplastic pemphigus showed reactivity with recombinant Dsc 1-3, Dsg 1, and Dsg3 protein (Figure 2B, lower panel).

\section{Purification of Dsc3-Reactive IgG from Pemphigus Sera}

Affinity chromatography columns were prepared with $\mathrm{CNBr}$ activated sepharose 4B (Amersham Biosciences) diluted in 1
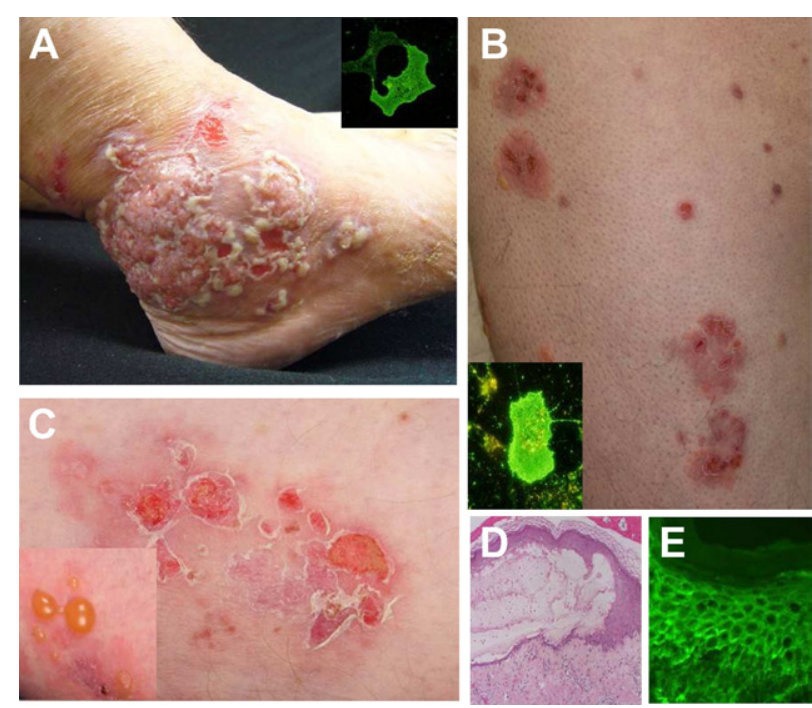

Figure 1. Clinical phenotype of representative patients with atypical pemphigus. Clinical pictures of two representative patients with atypical pemphigus (pemphigus vegetans and pemphigus herpetiformis), illustrating hypertrophic verrucous plaques with pustules and erosions of the foot (A) and multiple erythematous herpetiform erosions of the trunk and extremities $(\mathbf{B}, \mathbf{C})$. Insets of $\mathbf{A}$ and $\mathbf{B}$ show IgG reactivity of the patients' sera with human desmocollin 3-transfected COS-7 cells. Histopathology of lesional skin reveals intraepidermal loss of keratinocyte adhesion at the suprabasilar level (D). Direct immunofluorescence shows IgG deposits on the surface of epidermal keratinocytes (E). 
A

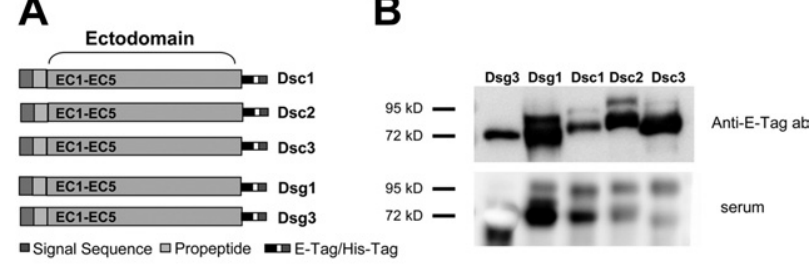

C

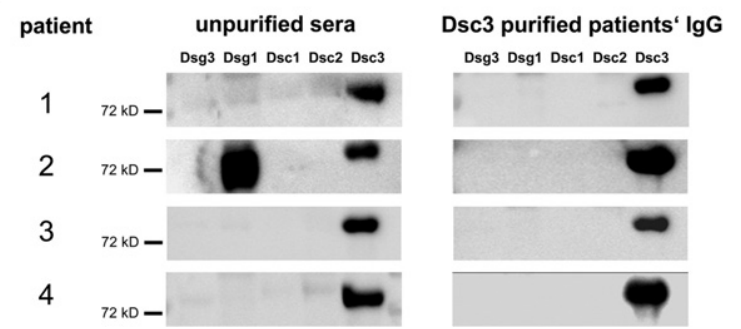

Figure 2. Affinity purification of desmocollin 3 (Dsc3)-reactive IgG autoantibodies from four patients with atypical pemphigus. A: Scheme of the recombinant proteins applied in this study, ie, the entire ectodomains of Dsc1, Dsc2, Dsc3, desmoglein 1 (Dsg1), and desmoglein 3 (Dsg3) linked to E- and 6xHisTag. B: Detection of the recombinant proteins by immunoblot analysis using an anti-E-tag monoclonal antibody (upper panel) or serum of a patient with paraneoplastic pemphigus (lower panel). C: Immunoblot analysis revealing IgG reactivity of the four atypical pemphigus sera, with the recombinant proteins before (left panels) and after (right panels) affinity purification, using Dsc3 columns. Patient 2 shows additional IgG reactivity with Dsg1.

$\mathrm{mmol} / \mathrm{L} \mathrm{HCl}$ as recommended by the manufacturer. Before coupling, the ligands (recombinant Dsc3 or Dsg3) were dialyzed against coupling buffer $\left(0.1 \mathrm{M} \mathrm{NaHCO}_{3}, 0.5 \mathrm{M} \mathrm{NaCl}, \mathrm{pH}\right.$ 8.3) and incubated with the sepharose medium overnight at $4^{\circ} \mathrm{C}$. The next day, the coupled sepharose was subjected to five washes with coupling buffer followed by incubation with blocking buffer $(0.1 \mathrm{M}$ Tris- $\mathrm{HCl}$ at $\mathrm{pH} 8.0)$ for 2 hours at room temperature. The coupled sepharose was then washed four times with $0.1 \mathrm{M}$ acetate and $0.5 \mathrm{M} \mathrm{NaCl}$ at $\mathrm{pH} 4.0$ and four times with $0.1 \mathrm{M} \mathrm{Tris-} \mathrm{HCl}$ and $0.5 \mathrm{M} \mathrm{NaCl}$ at $\mathrm{pH} 8.0$ alternately. Finally, the sepharose was washed with PBS containing 0.5 $\mathrm{mmol} / \mathrm{L} \mathrm{CaCl}_{2}$ at $\mathrm{pH} 7.4$.

Pemphigus sera were diluted at 1:5 in PBS containing 0.5 $\mathrm{mmol} / \mathrm{L} \mathrm{CaCl}$ at $\mathrm{pH}$ 7.4. The Dsc3 column was incubated with the diluted sera overnight at $4^{\circ} \mathrm{C}$. The next day, the pre-adsorbed sera were collected and the column was washed three times with PBS containing $0.5 \mathrm{mmol} / \mathrm{L} \mathrm{CaCl}_{2}+0.05 \%$ Tween 20 at $\mathrm{pH} 7.4$ and, subsequently, three times with PBS containing $0.5 \mathrm{mmol} / \mathrm{L} \mathrm{CaCl}_{2}$ at $\mathrm{pH}$ 7.4. The antibodies were eluted using $100 \mathrm{mmol} / \mathrm{L}$ Glycin- $\mathrm{HCl}(\mathrm{pH} 2.7)$, and $900 \mu \mathrm{L}$ aliquots were collected in tubes containing $100 \mu \mathrm{L}$ of $1 \mathrm{M}$ Tris- $\mathrm{HCl}(\mathrm{pH}$ 9.0). The antibodies containing aliquots were combined (total volume of $15 \mathrm{ml}$ ) and dialyzed overnight against PBS with 0.5 $\mathrm{mmol} / \mathrm{L} \mathrm{CaCl}_{2}$ at $\mathrm{pH}$ 7.4. The purified IgG fraction was concentrated 10-fold using Amicon Ultra-15 Centrifugal Filter Units (Millipore, Billerica, MA). The protein concentration of the eluate was measured using a modified Lowry protein assay. Final protein concentrations ranged between 100 and $200 \mu \mathrm{g} / \mathrm{ml}$.

\section{Immunoblot Analysis with Dsc and Dsg} Recombinants

The Dsg and Dsc recombinants were run on $10 \%$ SDSPAGE and blotted onto nitrocellulose membranes as previ- ously described. ${ }^{9,15}$ Membranes were blocked with 5\% milk powder in PBS, 0.05\% Tween-20 (PBS-T) with $0.5 \mathrm{mmol} / \mathrm{L}$ $\mathrm{CaCl}_{2}$. Pemphigus sera (1:100) and purified IgG fractions (1:20) were diluted in PBS-T with $0.5 \mathrm{mmol} / \mathrm{L} \mathrm{CaCl}_{2}$ and $5 \%$ milk powder and were incubated with the blotted membranes overnight at $4^{\circ} \mathrm{C}$. After three washes with PBS-T supplemented with $0.5 \mathrm{mmol} / \mathrm{L} \mathrm{CaCl}_{2}$, the nitrocellulose membranes were incubated with horseradish peroxidase (HRP)-conjugated anti-human IgG (1:5000; Dako, Glostrup, Denmark) for 1 hour at room temperature. Specific immunoreactivity was then visualized using Immobilon Western Chemiluminescent HRP substrate (Millipore) (Figure 2, B and C).

\section{Immunofluorescence Studies}

The immortalized keratinocyte cell line HaCaT was cultured in Dulbecco's modified Eagle's medium (DMEM high glucose; Invitrogen) supplemented with $10 \%$ fetal calf serum (PAA, Pasching, Austria), 50 units $/ \mathrm{ml}$ penicillin-G, $50 \mu \mathrm{g}$ streptomycin and $2 \mathrm{mmol} / \mathrm{L}$ L-Glutamine (Gibco, Karlsruhe, Germany). For immunofluorescence staining, $\mathrm{HaCaT}$ cells were grown subconfluent in chamber slides (Nunc/Thermo, Fisher Scientific, Wiesbaden, Germany) using epidermal keratinocyte medium (CnT-57 medium; CELLnTEC Advanced Cell Systems, Bern, Switzerland). Subconfluent keratinocyte cultures were switched to defined keratinocyte medium (CnT-02 medium; CELLnTEC Advanced Cell Systems) supplemented with $1.2 \mathrm{mmol} / \mathrm{L} \mathrm{CaCl}_{2} 24$ hours before adding the IgG fractions. Keratinocytes were then incubated for 2 hours at $4^{\circ} \mathrm{C}$ with purified anti-Dsc3 IgG diluted at 1:2, pooled IgG from healthy donors 1:2 diluted or antiDsc3 monoclonal antibodies (clone U114, $1 \mu \mathrm{g} / \mathrm{ml}$; Progen, Heidelberg, Germany). After three washes with PBS containing $1.2 \mathrm{mmol} / \mathrm{L} \mathrm{CaCl}_{2}$ and $1.0 \mathrm{mmol} / \mathrm{L} \mathrm{MgCl}_{2} \quad 6 \mathrm{H}_{2} \mathrm{O}$, keratinocytes were fixed in $1 \%$ paraformaldehyde-PBS for 20 minutes at room temperature. After three washes, cells were incubated with PBS containing $0.5 \%$ Triton X-100 for 10 minutes at room temperature and were finally incubated with fluorescein isothiocyanate (FITC)-labeled anti-human IgG at 1:200 in 1\% bovine serum albumin-PBS (dianova, Hamburg, Germany) (Figure 3A-F).

Frozen sections of normal human skin were blocked for 30 minutes with PBS supplemented with 10\% normal goat serum. Samples were incubated for 1 hour at room temperature with purified Dsc3-reactive IgG (diluted in PBS with $2 \%$ goat serum at $1: 2$ ), IgG pooled from healthy donors at the same dilution or anti-Dsc3 monoclonal IgG (U114; Progen) as positive control for Dsc3 reactivity. After three washes with PBS, the skin sections were incubated with FITC-labeled rabbit anti-human IgG (1:200; dianova) for 30 minutes at room temperature (Figure 3G-L).

Indirect immunofluorescence on monkey esophagus was performed following a standardized protocol according to the manufacturer's instructions (The Binding Site, Birmingham, UK). (Figure 3M-R).

\section{Dispase-Based Keratinocyte Dissociation Assay}

Primary human epidermal keratinocytes were seeded in 12-well plates and grown to confluence in CnT-57 medium 


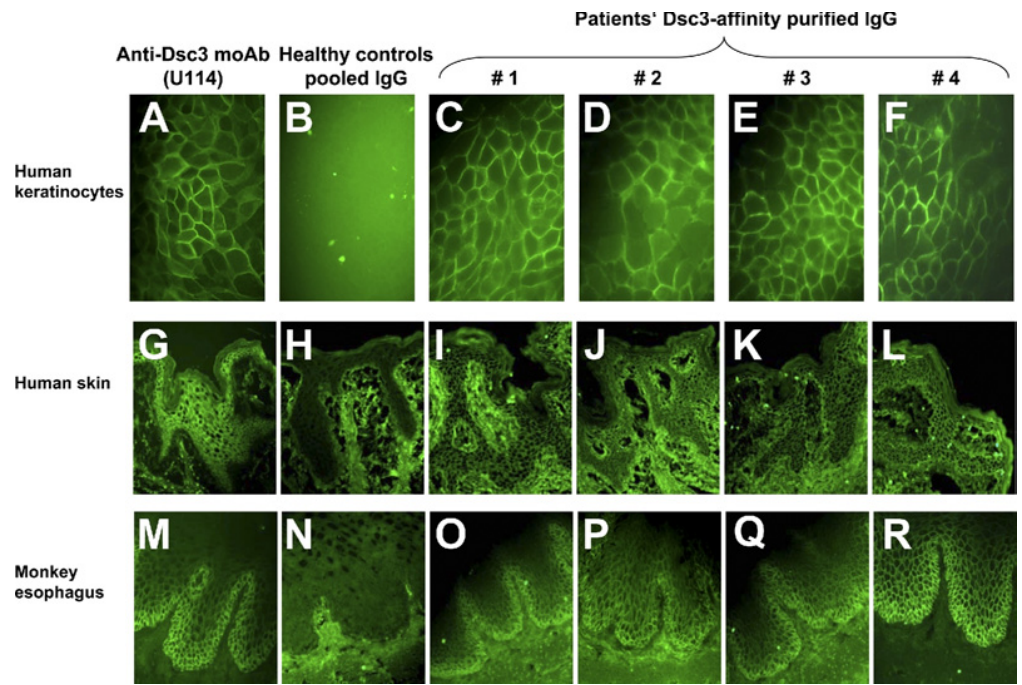

Figure 3. Reactivity of the purified desmocollin 3 (Dsc3)-reactive IgG autoantibodies with cultured human keratinocytes, human epidermis, and monkey esophagus. Dsc3 affinity-purified IgG from four patients, with atypical pemphigus, reveal intercellular reactivity with cultured human keratinocytes (HaCaT cells) [patients 1-4 (C-F)], human epidermis (I-L) and monkey esophagus (O-R). Monoclonal anti-Dsc3 antibody (clone U114) (A, G, and $\mathbf{M}$ ) and pooled IgG of healthy control sera ( $\mathbf{B}, \mathbf{H}$, and $\mathbf{N})$ served as positive and negative controls, respectively.

(CELLnTEC Advanced Cell Systems) as previously described. ${ }^{16}$ The day before the assay, cells were cultured in CnT-02 medium (CELLnTEC Advanced Cell Systems) containing $1.2 \mathrm{mmol} / \mathrm{L} \mathrm{CaCl}_{2}$ and incubated with purified Dsc3reactive IgG (at $20 \mu \mathrm{g} / \mathrm{ml})$, pooled control lgG (20 $\mu \mathrm{g} / \mathrm{ml})$ or anti-Dsc3 monoclonal antibody (U114 at $1 \mu \mathrm{g} / \mathrm{ml}$; Progen), overnight at $37^{\circ} \mathrm{C}$. Two hours before the assay termination, recombinant exfoliative toxin A (Toxin Technology, Sarasota, FL) was added at $0.5 \mu \mathrm{g} / \mathrm{ml}$ to cleave Dsg1 on the keratinocyte surface. After two washes with PBS, the adherent keratinocytes were incubated at $37^{\circ} \mathrm{C}$ for 20 minutes with dispase I (Roche Applied Sciences, Mannheim, Germany) resulting in a non-adherent cell monolayer. The monolayers were carefully washed twice with PBS and subjected to mechanical stress by pipetting ten times with a $1-\mathrm{ml}$ pipet. Fragments were fixed in $1 \mathrm{ml}$ of a $10 \%$ formalin solution and stained with crystal violet. Images were captured using a digital camera and fragments were counted by five different blinded observers (Figure 4). Relative dissociation scores were calculated using the number of keratinocyte fragments in relation to the maximal number of cell fragments obtained by the positive control (anti-dsc3 monoclonal antibody, clone U114).

\section{Results}

\section{Dsc3-Reactive Autoab in the Sera of Four Patients with Atypical Pemphigus}

Sera of four patients with pemphigus herpetiformis and pemphigus vegetans, respectively, were included in this study. Patients presented with multiple erythematous herpetiform erosions and with hypertrophic verrucous plaques with pustules and erosions, respectively, which are illustrated in Figure 1. Histopathology analysis of le-
A

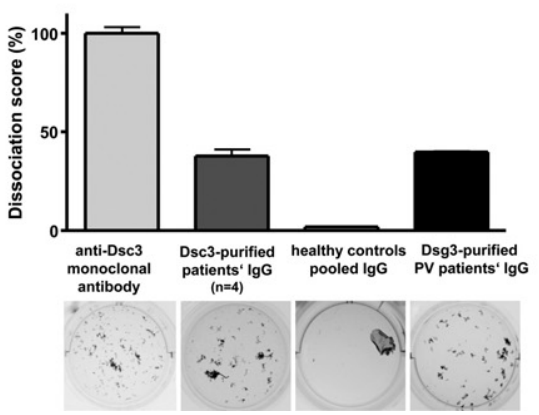

B

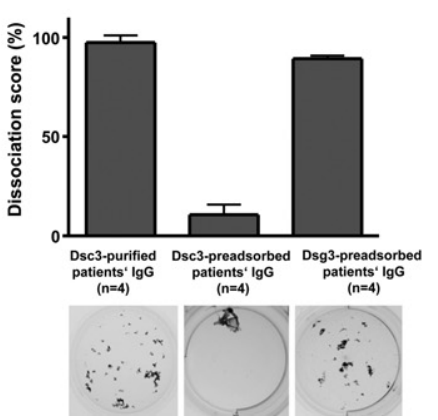

C

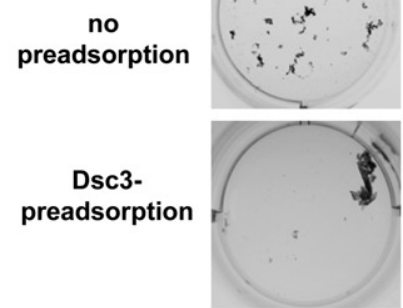

Dsg3-purified

PV patients' IgG

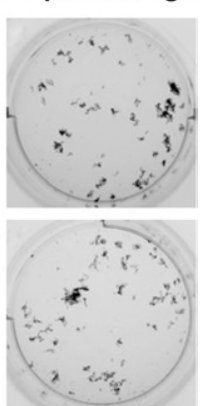

Figure 4. Desmocollin 3 (Dsc3)-affinity purified IgG from four patients with atypical pemphigus induces loss of keratinocyte adhesion. Results of the dispase-based keratinocyte dissociation assay using primary human epidermal keratinocytes are illustrated. Keratinocyte monolayers were incubated with the monoclonal anti-Dsc3 IgG antibody (clone U114), Dsc3-affinity purified IgG from the four atypical pemphigus patients, pooled IgG from healthy donors, or desmoglein 3 (Dsg3)-affinity purified IgG from a pemphigus vulgaris patient. A: Quantification of the keratinocyte fragments is depicted as a relative dissociation score. The maximal number of keratinocyte fragments obtained after incubation with the monoclonal anti-Dsc3 IgG antibody (clone U114) is set as $100 \%$. Representative images out of three independent experiments are shown below the diagram. B: Number of keratinocyte fragments after incubation with Dsc3-affinity purified IgG from four atypical pemphigus patients is illustrated as a dissociation score of 100\% (B, left column). The induction of keratinocyte dissociation is Dsc3-specific because pre-adsorption of the Dsc3-affinity purified IgG fraction with recombinant Dsc3 (B, middle column and $\mathbf{C}$, left panel) but not with human Dsg3 (B, right column) blocks keratinocyte dissociation. To exclude non-specific binding of IgG to recombinant Dsc3 protein, Dsg3-purified IgG from a pemphigus vulgaris patient was pre-adsorbed with Dsc3 protein (C, right panels). Dsg3 affinity purified IgG induces loss of keratinocyte adhesion (A and $\mathbf{C})$ that is not inhibited by pre-adsorption with human Dsc3 protein (C, right panels). 
sional skin indicated intraepidermal loss of keratinocyte adhesion at the suprabasilar layer (Figure 1D). Furthermore, direct immunofluorescence revealed an intercellular staining pattern, ie, positive IgG deposits on the surface of epidermal keratinocytes (Figure 1E).

To identify the desmosomal target proteins recognized by the IgG autoab of these patients, the sera were reacted with defined baculovirus-derived proteins including Dsg1, Dsg3, and Dsc1-39,15 (Figure 2, A and B, upper panel). Immunoblot analysis revealed the presence of IgG against Dsc3 in all of the studied patients; in addition, the serum of patient 2 showed IgG reactivity against Dsg1 (Figure 2C, left panel; Table 1).

\section{Dsc3-Specific lgG Recognizes Recombinant and Native Dsc3}

Serum samples of four atypical pemphigus patients were affinity purified using a Dsc3-coupled-CNBr sepharose column to obtain and further analyze the Dsc3-reactive IgG fraction. After purification, the $\mathrm{IgG}$ fractions of the four studied patients reacted exclusively with recombinant Dsc3, which was indicated by immunoblot analysis (Figure 2C, right panels). To determine the reactivity of the Dsc3-purified IgG also with native Dsc3, immunofluorescence of cultured human keratinocytes (HaCat) and human skin samples was also performed (Figure 3). The anti-Dsc3 monoclonal antibody U114 was used as a positive control and showed intercellular staining of both keratinocyte cell surface (Figure $3 A$ ) and human epidermis (Figure 3G), whereas pooled IgG from healthy control sera did not show any specific staining (negative control, Figure $3 \mathrm{~B}, \mathrm{H}$ ). The Dsc3-purified IgG fractions of patients 1-4 demonstrated intercellular reactivity with cultured human keratinocytes (Figure 3C-F) and human epidermis (Figure 3I-L). In addition, indirect immunofluorescence was performed on monkey esophagus confirming the previous results (Figure 3M-R). The anti-Dsc3 monoclonal antibody, U114, elicited an intercellular staining pattern predominantly at the expected basal and suprabasal levels of the mucosal epithelium (Figure 3M). Very similar results were obtained subjecting the Dsc3-purified IgG fractions of patients 1-4 to immunofluorescent staining on monkey esophagus (Figure 3O-R), demonstrating the binding of these Dsc3-purified autoab to native Dsc3.

\section{Dsc3-Specific IgG Induces Loss of Keratinocyte Adhesion in Vitro}

After confirming the reactivity of Dsc3-purified IgG autoab with recombinant and native Dsc3, we sought to investigate the pathogenic relevance of these autoab. Interference with keratinocyte adhesion is considered the pathogenic hallmark of autoab in pemphigus. Thus, we applied a well established in vitro system for evaluating the capability of Dsc3-purified IgG autoab to induce loss of keratinocyte adhesion. ${ }^{16}$ Dsc3-purified IgG of pemphigus patients 1-4 was applied to a dispase-based keratinocyte dissociation assay using primary human keratinocytes. Confluently grown primary keratinocytes were incubated with Dsc3- purified IgG fractions of patients 1-4, and afterwards Dsg1 was degraded by incubation with exfoliatoxin A. Next, mechanical stress was applied to the keratinocyte monolayer to obtain cellular fragments illustrating the loss of keratinocyte adhesion in these keratinocyte cultures and thus, the pathogenicity of the Dsc3 autoab studied. Figure 4 illustrates that the anti-Dsc3 monoclonal antibody, U114, induces a dramatic weakening of keratinocyte adhesion, leading to a high number of keratinocyte fragments, whereas control IgG from pooled healthy individuals does not affect keratinocyte adhesion (Figure 4A). Keratinocyte cell sheets incubated with Dsc3-purified IgG were dissociated into numerous smaller fragments, indicating that Dsc3reactive IgG of patients' sera is capable of inducing loss of keratinocyte adhesion (Figure 4A).

Furthermore, it was crucial to investigate whether this effect on keratinocyte adhesion was specific for the Dsc3reactive autoab and related to the presence of Dsc3 in desmosomes. Pre-adsorption of the Dsc3-purified IgG fractions with recombinant Dsc3, but not with recombinant Dsg3, completely blocked the loss of keratinocyte adhesion in the dissociation assay (Figure 4B). To exclude non-specific binding of IgG autoab to recombinant Dsc3, we also applied Dsg3-purified IgG from PV patients to the keratinocyte dissociation assay. As previously described, Dsg3reactive autoab of a pemphigus vulgaris patient induces loss of keratinocyte adhesion in this assay (Figure 4, A and C). However, pre-incubation of anti-Dsg3 IgG with recombinant Dsc3 protein did not abrogate this effect (Figure 4C, right panel). Thus, these results strongly suggest that the Dsc3-purified IgG fraction in the atypical pemphigus patients studied here is pathogenic and induces loss of keratinocyte adhesion by specifically binding and/or blocking Dsc3 and such effect can be blocked by preincubation with the recombinant Dsc3-protein, but not with Dsg3.

\section{Discussion}

This study presents that Dsc3-reactive autoab from patients with atypical pemphigus induce loss of epidermal keratinocyte adhesion. The affinity-purified Dsc3 autoab from the four atypical pemphigus patients induced loss of cellular adhesion in a dispase-based keratinocyte dissociation assay. These results extend recent findings of Spindler et al, ${ }^{14}$ which identified Dsc3 as a crucial component of desmosomal adhesion. Moreover, they reported that epidermal adhesion mediated by Dsc3 is inhibited by sera from a subset of patients with atypical pemphigus. ${ }^{14}$ IgG autoab against Dsc3 are not exclusively associated with atypical pemphigus; we and others have also identified IgG reactivity against Dsg1 and Dsg3 in single cases of pemphigus herpetiformis. ${ }^{17,18}$

The best evidence that Dsc3 is critically involved in desmosomal adhesion in the epidermis comes from a recently established $D s c 3^{-1-}$ mouse model. ${ }^{13}$ The $D s c 3^{-1-}$ mice revealed an impressive phenotype with extensive intraepidermal blistering and telogen hair loss. This phenotype shares some similarities with a mouse deficient of Dsg3, the autoantigen of PV. ${ }^{19}$ Thus, in addition to Dsg3 and Dsg1, 
Dsc3 must be considered as a relevant component for desmosomal adhesion of epidermal keratinocytes.

Until now, there was only circumstantial evidence for a pathogenic role of IgG autoab against distinct Dsc isoforms. Dsc1 has been previously identified as a target antigen of IgA autoab in the subcorneal pustular dermatosis type of IgA pemphigus. ${ }^{8,20}$ Except for the findings of a single in vitro study, ${ }^{21}$ there is currently no clear evidence for the pathogenicity of IgA autoab on desmosomal cell-cell adhesion. Autoab against Dsc of the IgG and IgA classes have been occasionally identified in patients with atypical pemphigus $^{9,10-12,17}$ and paraneoplastic pemphigus. ${ }^{9,22,23}$

It is currently a matter of debate whether IgG or IgA autoab against Dsc are rare phenomena in pemphigus or whether their detection is limited by the diagnostic tools that are currently available. Hisamatsu et al ${ }^{11}$ did not detect lgG or IgA autoab against Dsc1-3 in 45 sera from classical pemphigus patients by enzyme-linked immunosorbent assay (ELISA) with recombinant proteins. The sensitivity of the Dsc-ELISA was limited because all of the sera from eight patients with IgA pemphigus revealed IgA reactivity with Dsc1 expressed on COS cells, but only one serum was IgA positive by ELISA. ${ }^{11}$ Using baculovirus-derived, eukaryotic recombinants of Dsc1, Dsc2, and Dsc3, Müller et al ${ }^{9}$ were not able to detect Dsc-specific lgG in the sera of a cohort of 74 European patients with PV by ELISA and immunoblot, respectively. However, a considerable number of sera from patients with atypical forms of pemphigus, paraneoplastic pemphigus, and IgA pemphigus demonstrated either IgG or IgA reactivity with Dsc1, Dsc2, or Dsc3. ${ }^{9}$ This observation is also reflected by the findings of the present study that the four Dsc3-reactive sera studied here were derived from patients with atypical pemphigus (pemphigus vegetans and pemphigus herpetiformis) who presented with cutaneous blisters; additional IgG reactivity against Dsg1 was found in only one case. Thus, the low detection rate of Dsc-reactive IgG autoab in PV and PF is presumably not a technical artifact, rather it is due to the low prevalence of Dsc-reactive autoab in PV or PF.

In summary, the present findings demonstrate that lgG autoab against Dsc3 induce loss of keratinocyte adhesion, which strongly suggests their pathogenic relevance in pemphigus. Because Dsc3-reactive IgG is only rarely found in patients with classical PV or PF, the observed in vitro pathogenicity of anti-Dsc3 IgG autoab provides a sound explanation why Dsc3-reactive patients with atypical pemphigus lacking IgG against Dsg3 or Dsg1 develop skin blistering.

\section{Acknowledgments}

We are grateful to Eva Podstawa, Nadine Losse, and Andrea Gerber for excellent technical assistance.

\section{References}

1. Amagai M, Klaus-Kovtun V, Stanley JR: Autoantibodies against a novel epithelial cadherin in pemphigus vulgaris, a disease of cell adhesion. Cell 1991, 67:869-877

2. Hertl M, Eming R, Veldman C: T cell control in autoimmune bullous skin disorders. J Clin Invest 2006, 116:1159-1166
3. Amagai M, Hashimoto T, Shimizu N, Nishikawa T: Absorption of pathogenic autoantibodies by the extracellular domain of pemphigus vulgaris antigen (Dsg3) produced by baculovirus. J Clin Invest 1994, 94:59-67

4. Mahoney MG, Wang Z, Rothenberger K, Koch PJ, Amagai M, Stanley JR: Explanations for the clinical and microscopic localization of lesions in pemphigus foliaceus and vulgaris. J Clin Invest 1999, 103: 461-468

5. Amagai M, Koch PJ, Nishikawa T, Stanley JR: Pemphigus vulgaris antigen (desmoglein 3) is localized in the lower epidermis, the site of blister formation in patients. J Invest Dermatol 1996, 106:351-355

6. Green KJ, Simpson CL: Desmosomes: new perspectives on a classic. J Invest Dermatol 2007, 127:2499-2515

7. Cheng X, Den Z, Koch PJ: Desmosomal cell adhesion in mammalian development. Eur J Cell Biol 2005, 84:215-223

8. Hashimoto T, Kiyokawa C, Mori O, Miyasato M, Chidgey MA, Garrod DR, Kobayashi Y, Komori K, Ishii K, Amagai M, Nishikawa T: Human desmocollin 1 (Dsc1) is an autoantigen for the subcorneal pustular dermatosis type of IgA pemphigus. J Invest Dermatol 1997, 109:127-131

9. Müller R, Heber B, Hashimoto T, Messer G, Mullegger R, Niedermeier A, Hertl M: Autoantibodies against desmocollins in European patients with pemphigus. Clin Exp Dermatol 2009, 34:898-903

10. Hashimoto T, Amagai M, Watanabe K, Dmochowski M, Chidgey MA, Yue KK, Garrod DR, Nishikawa T: A case of pemphigus vulgaris showing reactivity with pemphigus antigens (Dsg1 and Dsg3) and desmocollins. J Invest Dermatol 1995, 104:541-544

11. Hisamatsu $Y$, Amagai M, Garrod DR, Kanzaki T, Hashimoto T: The detection of $\lg$ and $\lg A$ autoantibodies to desmocollins 1-3 by enzyme-linked immunosorbent assays using baculovirus-expressed proteins, in atypical pemphigus but not in typical pemphigus. $\mathrm{Br} J$ Dermatol 2004, 151:73-83

12. Dmochowski M, Hashimoto T, Chidgey MA, Yue KK, Wilkinson RW, Nishikawa T, Garrod DR: Demonstration of antibodies to bovine desmocollin isoforms in certain pemphigus sera. Br J Dermatol 1995 , 133:519-525

13. Chen J, Den Z, Koch PJ: Loss of desmocollin 3 in mice leads to epidermal blistering. J Cell Sci 2008, 121:2844-2849

14. Spindler V, Heupel WM, Efthymiadis A, Schmidt E, Eming R, Rankl C Hinterdorfer P, Muller T, Drenckhahn D, Waschke J: Desmocollin 3-mediated binding is crucial for keratinocyte cohesion and is impaired in pemphigus. J Biol Chem 2009, 284:30556-30564

15. Muller R, Svoboda V, Wenzel E, Muller HH, Hertl M: IgG against extracellular subdomains of desmoglein 3 relates to clinical phenotype of pemphigus vulgaris. Exp Dermatol 2008, 17:35-43

16. Ishii K, Harada R, Matsuo I, Shirakata Y, Hashimoto K, Amagai M: In vitro keratinocyte dissociation assay for evaluation of the pathogenicity of anti-desmoglein $3 \mathrm{lgG}$ autoantibodies in pemphigus vulgaris. J Invest Dermatol 2005, 124:939-946

17. Kozlowska A, Hashimoto T, Jarzabek-Chorzelska M, Amagai A, Nagata $Y$, Strasz Z, Jablonska S: Pemphigus herpetiformis with IgA and IgG antibodies to desmoglein 1 and IgG antibodies to desmocollin 3. J Am Acad Dermatol 2003, 48:117-122

18. Lebeau S, Müller R, Masouyé I, Hertl M, Borradori L: Pemphigus herpetiformis: analysis of the autoantibody profile during the disease course with changes in the clinical phenotype. Clin Exp Dermatol 2010, 35:366-372

19. Koch PJ, Mahoney MG, Ishikawa H, Pulkkinen L, Uitto J, Shultz L, Murphy GF, Whitaker-Menezes D, Stanley JR: Targeted disruption of the pemphigus vulgaris antigen (desmoglein 3 ) gene in mice causes loss of keratinocyte cell adhesion with a phenotype similar to pemphigus vulgaris. J Cell Biol 1997, 137:1091-1102

20. Heng A, Nwaneshiudu A, Hashimoto T, Amagai M, Stanley JR: Intraepidermal neutrophilic $\mathrm{IgA} / \mathrm{lgG}$ antidesmocollin 1 pemphigus. $\mathrm{Br} \mathrm{J}$ Dermatol 2006, 154:1018-1020

21. Supapannachart N, Mutasim DF: The distribution of IgA pemphigus antigen in human skin and the role of IgA anti-cell surface antibodies in the induction of intraepidermal acantholysis. Arch Dermatol 1993, 129:605-608

22. Chorzelski TP, Hashimoto T, Nishikawa T, Ebihara T, Dmochowski M, Ismail M, Jablonska S: Unusual acantholytic bullous dermatosis associated with neoplasia and IgG and IgA antibodies against bovine desmocollins I and II. J Am Acad Dermatol 1994, 31:351-355

23. Hashimoto T: Immunopathology of paraneoplastic pemphigus. Clin Dermatol 2001, 19:675-682 